\title{
CREEP ANISOTROPY IN THE MONOCRYSTALLINE NICKEL-BASE SUPERALLOY CMSX-4
}

\author{
V. Sass, U. Glatzel and M. Feller-Kniepmeier \\ Technische Universitaet Berlin, Institut fuer Metallforschung, \\ Sekr. BH 18, Str. des 17. Juni 135 , \\ D10623 Berlin, Germany
}

\begin{abstract}
$\underline{\text { bstract }}$
The orientation dependence of creep strength of the single crystal superalloy CMSX-4 was studied in the temperature range $1123-1253 \mathrm{~K}$. At $1123 \mathrm{~K}$ creep behavior and lifetimes for small strains are highly anisotropic with the highest variations occurring in the primary creep regime. Secondary creep strength decreases in the order [001] - [011] - [111]. Under the present conditions plastic deformation in the primary and at the beginning of secondary creep stage occurs mainly in the matrix phase. The motion of matrix dislocations is strongly influenced by coherency stresses, which restrict dislocations from entering certain types of matrix channels and control the number of active slip systems. Extensive cutting of the $\gamma^{\prime}$-phase is observed only in [011] oriented samples and in [001] orientation under the highest applied stress and results in pronounced primary creep. The poor creep strength of [111] oriented crystals is related to poor work hardening and a cross slip mechanism of matrix screw dislocations.

At the higher temperature of $1253 \mathrm{~K}$ creep behavior is affected by the instability of the $\gamma^{\prime}$-morphology and the extent of anisotropy is highly reduced. The creep strength of the [ī11] orientation, however, remains poor.
\end{abstract}

\section{Introduction}

In order to take full advantage of the high temperature capabilities of single crystal superalloys, the strong anisotropy of various properties including high temperature creep strength must be considered. Although turbine blades are mainly stressed in the [001] direction, which is their natural solidification direction, their complex shapes and high temperature gradients produce locally multiaxial stress states and high thermal stresses acting in various directions. A comprehensive study of the influence of orientation on the creep behavior of the single crystal alloys MAR-M200 and MAR-M247 at $1047 \mathrm{~K}$ was presented by MacKay and Maier [1]. They reported long lifetimes for orientations near [i11] or [001] and short lives for samples oriented close to [011]. The creep strength was found to depend mainly on the amount of lattice rotation required to produce intersecting

$$
\text { Superalloys } 1996
$$

Edited by R. D. Kissinger, D. J. Deye, D. L. Anton,

A. D. Cetel, M. V. Nathal, T. M. Pollock, and D. A. Woodford

The Minerals, Mctals \& Matcrials Socicty, 1996 slip on $\{111\}<112>$ type slip systems, which is necessary for the transition from primary to secondary creep.

Caron et al. [2] showed for some advanced superalloys (CMSX-2, Alloy 454, MXON, CMSX-4) the strong influence of the $\gamma^{\prime}$-particle size on creep strength and rupture lives at intermediate temperatures $(1033 \mathrm{~K}-1123 \mathrm{~K})$. For a mean particle size of about $0.45 \mu \mathrm{m}$ optimum creep strength is obtained from orientations close to [001], whereas rupture lives for $[\overline{1} 11]$ are drastically reduced. For more elevated temperatures they reported a rapid decrease of the creep anisotropy due to a change in the prevailing deformation mechanism from heterogeneous cooperative shearing of the $\gamma / \gamma^{\prime}$-structure by $\{111\}<112>$ slip to a more homogenous $\{111\}<110>$ type slip in the matrix phase and creep induced morphology changes of the $\gamma^{\prime}$-phase. The authors also suggested, that the rhenium content of second generation superalloys may reduce the extent of anisotropic creep, however, no microscopic reasons for this behavior were given.

Generally most studies of creep anisotropy were mainly performed under testing conditions, which promoted extensive shearing of the $\gamma$-phase by $\{111\}<112>$ slip [1-4]. This mode of deformation results in a high degrec of anisotropy especially in the primary creep stage and accordingly in lifetimes for small strains. The transition to more homogenous matrix deformation with increasing temperature and decreasing stress tends to reduce orientation dependence [2]. At temperatures above 1173K, however, the instability of the $\gamma^{\prime}$-morphology makes it difficult to distinguish between the effects of different deformation mechanisms and raft formation. Unfortunately there is a lack of creep data and knowledge of deformation mechanisms in the temperature range between $1073 \mathrm{~K}$ and $1173 \mathrm{~K}$, where extensive shearing of the $\gamma^{\prime}$-phase disappears and the $\gamma^{\prime}$-morphology remains stable during creep tests.

Therefore the main target of this paper is the investigation of creep anisotropy in the advanced Re-containing alloy CMSX-4 at a temperature of $1123 \mathrm{~K}$. Analysis of creep data was focused on small strains up to $5 \%$ in order to study the creep behavior in primary and secondary creep stage, which are most relevant for technical applications. Additional tests at $1253 \mathrm{~K}$ were carried out to explore the influence of 
Table I: Nominal Chemical Composition of CMSX-4 (wt.-\%) [5].

\begin{tabular}{|c|c|c|c|c|c|c|c|c|c|}
\hline $\mathrm{Cr}_{\mathrm{r}}$ & $\mathrm{Al}$ & $\mathrm{Ti}$ & $\mathrm{Mo}$ & $\mathrm{W}$ & $\mathrm{Ta}$ & $\mathrm{Co}$ & $\mathrm{Re}$ & $\mathrm{Hf}$ & $\mathrm{Ni}$ \\
\hline 6.5 & 5.6 & 1.0 & 0.6 & 6.0 & 6.0 & 10.0 & 3.0 & 0.1 & bal. \\
\hline
\end{tabular}

morphological changes of the $\gamma^{\prime}$-phase on creep behavior. Extensive transmission electron microscopy (TEM) investigations were performed in order to identify the microscopic deformation mechanisms as function of crystal orientation and to provide an explanation for the macroscopic bchavior.

\section{Material and Experimental Procedures}

The chemical composition of the superalloy CMSX-4 is given in Table I. The addition of approximately $3 \mathrm{wt} . \%$ rhenium improves the creep strength of the material significantly $[5,6]$.

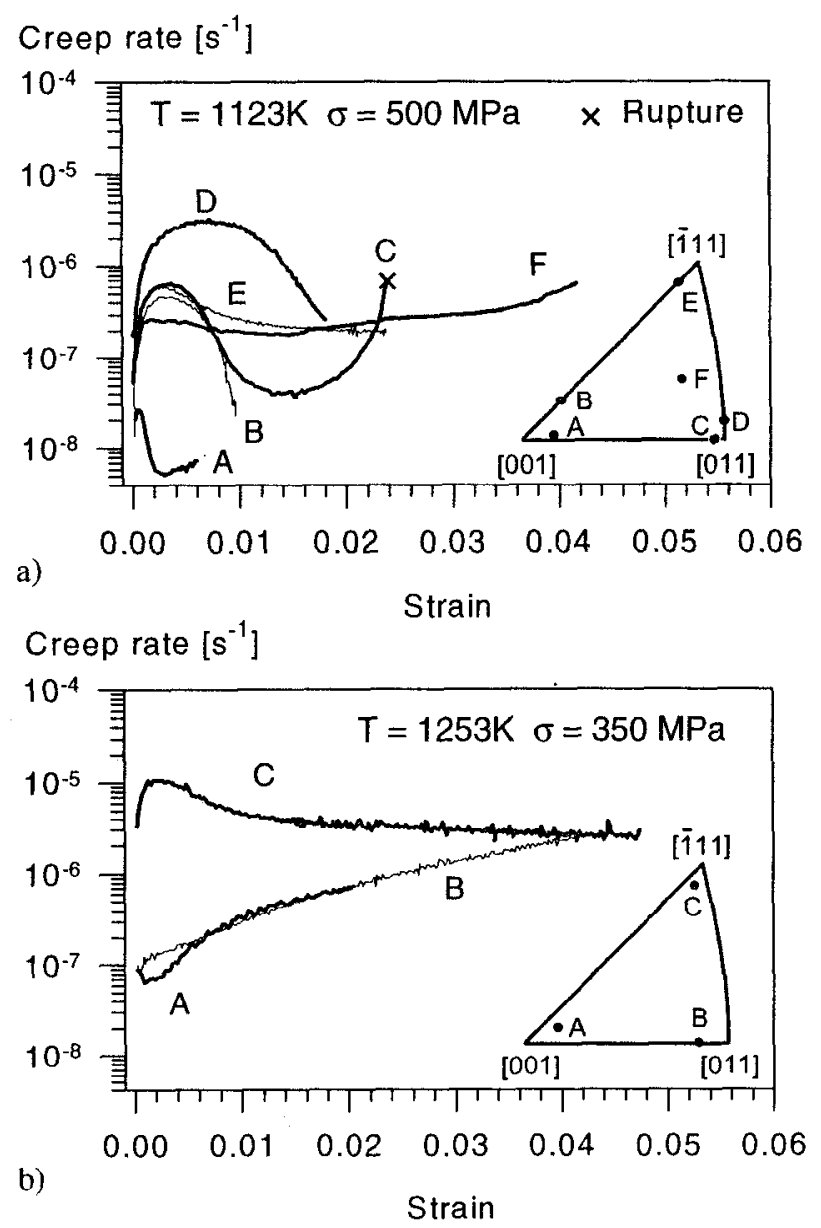

Figure 1: Typical creep curves of CMSX-4 single crystals for the main orientations plotted as strain rate $\dot{\varepsilon}$ vs. plastic strain $\varepsilon$. The exact orientations are given in the stereographic standard triangles. a) $\mathrm{T}=1123 \mathrm{~K}$, $\sigma=500 \mathrm{MPa}$. b) $\mathrm{T}=1253 \mathrm{~K}, \sigma=350 \mathrm{MPa}$.
Single crystal rods were grown using the withdrawal process with a selector technique for [001] orientations by Thyssen (Bochum) and a seeding technique for other orientations by VIAM (Moscow). All specimens received a standard heat treatment comprising a three-step solution treatment $(\mathrm{l} / 1553 \mathrm{~K}, 2 \mathrm{~h} / 1563 \mathrm{~K}, 6 \mathrm{~h} / 1573 \mathrm{~K})$ and a two step aging treatment $(6 \mathrm{~h} / 1413 \mathrm{~K}, 16 \mathrm{~h} / 1143 \mathrm{~K})$. The heat treatment produced cuhoidal $\gamma^{\prime}$-particles with a mean edge length of about $0.45 \mu \mathrm{m}$ taking up a volume fraction of about $70 \%$ [7]. A careful examination of the fully heat treated microstructures by scanning electron microscopy (SEM) and TEM showed no significant differences between crystals produced by the selector and the seeding technique.

Crecp specimens with a gauge length of $55 \mathrm{~mm}$ and a diameter of $9 \mathrm{~mm}$ for [001] oriented crystals and a gauge length of $62 \mathrm{~mm}$ and a diameter of $6 \mathrm{~mm}$ for other orientations were prepared by mechanical machining and grinding. The creep tests were performed in air under constant loads ranging from $350 \mathrm{MPa}$ to $650 \mathrm{MPa}$ at $1123 \mathrm{~K}$ and $1253 \mathrm{~K}$. The strain was measured continuously with extensometers. The creep tests were usually interrupted in different creep stages before rupture. The specimens were then cooled under load in order to preserve the typical microstructure for subsequent TEM-investigations. TEM analysis was carried out using a Philips CM 30 microscope operated at $300 \mathrm{kV}$ and a Jeol $200 \mathrm{C}$ microscope operated at $200 \mathrm{kV}$. The initial orientation of each single crystal was determined by the Laue back-reflection $\mathrm{X}$-ray technique.

\section{$\underline{\text { Results }}$}

Tensile creep test were performed in order to investigate the creep strength of CMSX-4 single crystals as a function of orientation, stress and temperature. At an intermediate temperature of $1123 \mathrm{~K}$ lifetimes for smaller strains were found to be governed by the primary creep behavior, which is highly sensitive to the crystal orientation. Typical creep curves for the main orientations under a load of $500 \mathrm{MPa}$ plotted as strain rate versus strain are shown in Fig. 1a. Although the shape of the creep curves is similar for all orientations, the strain rates and the amount of primary creep strain vary dramatically.

Pronounced primary creep is observed for all crystals except those close to the [001] orientation with a misorientation towards [011] (e.g. sample A). No clear transition from primary to secondary creep is visible for crystals oriented near $[\overline{1} 11]$ and in the center of the stercographic triangle. This is reflected in the strain-lifetimes for $0.5 \%$ and $2.0 \%$ plastic strain given in Fig. 2a. Creep strength decreases strongly in the order [001] - [011] - [T11].

The highest strain-lifetimes are obtained by near [001] oriented samples due to the small amplitude of primary 

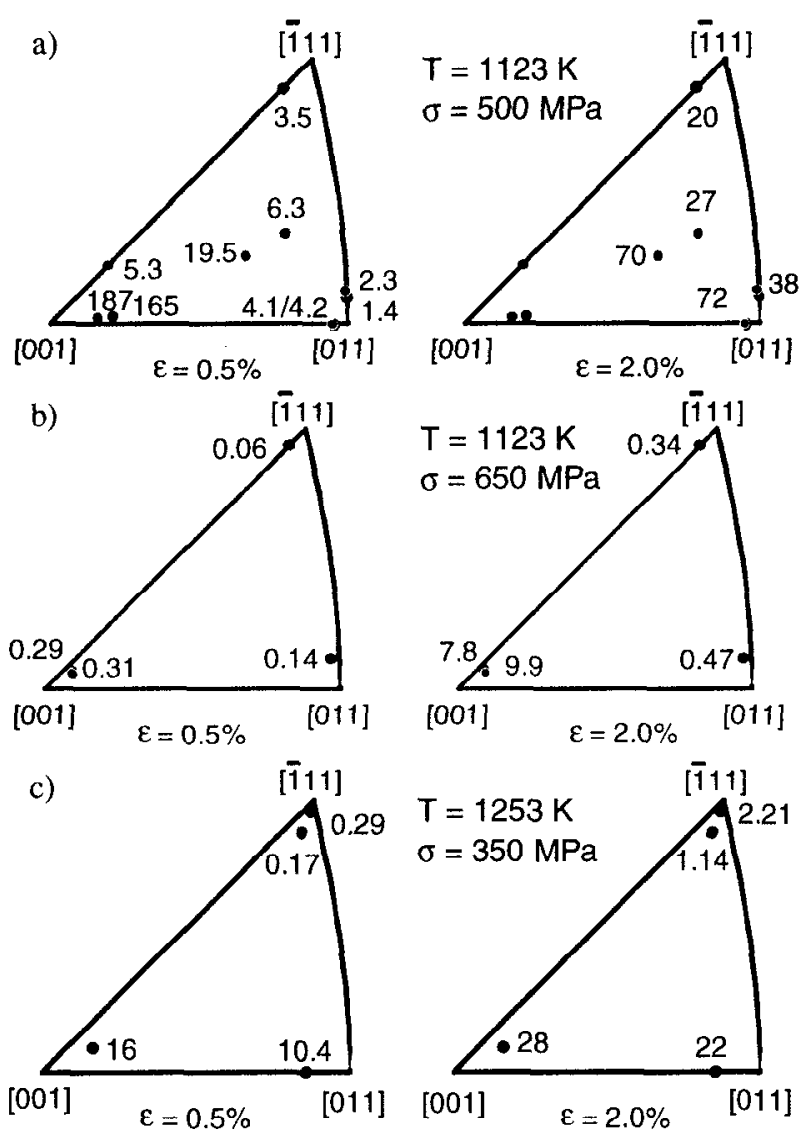

Figure 2: Strain lifetimes of CMSX-4 single crystals for 0.5 and $2.0 \%$ strain in hours plotted as function of orientation. a) $\mathrm{T}=1123 \mathrm{~K}$ and $\sigma=500 \mathrm{MPa}, \mathrm{b}) \mathrm{T}=1123 \mathrm{~K}$ and $\sigma=650 \mathrm{MPa}, \mathrm{b}) \mathrm{T}=1253 \mathrm{~K}$ and $\sigma=350 \mathrm{MPa}$.

creep and the lowest secondary creep rates. All other samples needed much larger strains to reach the secondary creep stage which explains the extremely short lifetimes for $0.5 \%$ strain.

At higher strains the secondary creep rates become more important and accordingly the lifetimes for the [111] orientation are the poorest. The lifetimes of crystals oriented near [001] and [011] are highly sensitive to misorientations away from the [001]-[011] boundary, which is a single slip orientation for $\{111\}<112>$ slip. Increasing the load to $650 \mathrm{MPa}$ leads to very similar results shown in Fig. $2 \mathrm{~b}$.

The effect of temperature on creep anisotropy is much more pronounced than the influence of stress. At a temperature of $1253 \mathrm{~K}$ and a load of $350 \mathrm{MPa}$ the creep curves for [001] and [011] orientations (Fig. 1b) have an altered shape with an overall minimum of the creep rates at small strains followed by a continuos softening with increasing strain. The same behavior has previously been reported to be typical for [001] oriented CMSX-4 samples at high temperatures [8]. The very similar lifetimes in Fig. $2 \mathrm{c}$ confirm, that the anisotropy between [001] and [011] virtually disappears at high temperatures. For [ $\overline{1} 11]$ the characteristic shape of the creep curve at lower temperatures with a pronounced maximum of the creep rate is preserved leading to very poor lifetimes for small strains.

\section{Discussion}

The creep tests of CMSX-4 single crystals showed a high degree of anisotropy, which is retained up to high tempcratures. The most prominent features of creep in CMSX-4 are the concentration of plastic deformation in the matrix phase at the smaller strains investigated here and the poor creep resistance of [1111] oriented crystals at both temperatures of $1123 \mathrm{~K}$ and $1253 \mathrm{~K}$. This has not been reported for other alloys [2]. The comparison of our result with other findings, however, is not straightforward, since we chose to evaluate creep strength by strain-lifetimes instead of total rupture lives. In the latter case the results depend strongly on rupture elongation and tertiary creep behavior, which may possess a different orientation dependence than the creep behavior in the prinlary and secondary creep stage we were interested in. Strain-lifetimes for small strains depend strongly on the primary creep behavior, which proved to be far more anisotropic at $1123 \mathrm{~K}$ than secondary creep behavior. It is therefore convenient to discuss both regimes separately.

\section{Primary Creep at $1123 \mathrm{~K}$}

The strong sensitivity of the primary creep behavior of crystals in [001] and [011] orientation to small misorientations is generally explained by the multiplicity of active $\{111\}<112>$ slip systems $[1,3,4]$. In this mode of deformation the $\gamma^{\prime}$-precipitates are sheared by $a / 3<112>$ partial dislocations under formation of a superlattice stacking faults (SSF). Single slip orientations promote high primary creep rates and strains due to the absence of strain hardening. Under the present conditions extensive $\{111\}<112>$ slip was observed only for [001] at $650 \mathrm{MPa}$ and particularly for [011] at $500 \mathrm{MPa}$ as shown in Fig. 4b,c. In the latter case misorientations towards [111] promote coplanar slip on the (111) slip plane as shown in Fig. 4b and result in exceedingly high primary creep rates (see sample D in Fig. 1a). For crystals oriented on the [001]-[011] boundary (e.g. sample C in Fig. 1a), which is a duplex slip orientation for $\{111\}<112>$ slip, intersecting slip on two \{111\} slip planes prevails from the start of plastic deformation on as can be seen from Fig. 4c. Here primary creep rates and strains are strongly reduced.

The predominant occurrence of $\gamma^{\prime}$-cutting in [011] orientation indicates, that the shear stresses required for the generation of superlattice stacking faults are higher for [001] than for [011], since the Schmid factors for $\{111\}<112>$ slip are equal in both cases. TEM investigations revealed, that in [011] cutting of the $\gamma^{\prime}$-phase occurs by a specific mechanism shown in Fig. 3 .

Two dipoles of $\{111\}<110>$ matrix dislocations marked as 1 and 2 expand in a $(001)$ matrix channel on the $(\overline{1} 11)$ plane. 


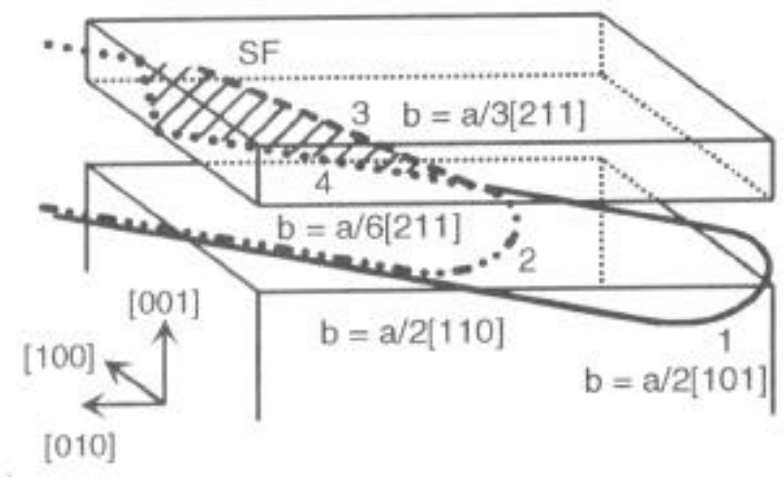

a)

Figure 3: Generation of stacking faults inside $\gamma^{\prime}$-particles in [011] oriented crystals by the reaction of two matrix dislocation loops, a) Schematic sketch. b) TEM micrograph taken after $0.14 \%$ strain at $\mathrm{T}=1123 \mathrm{~K}$ and $\sigma=500 \mathrm{MPa}$. The arrow marks the location of the dislocation reaction.

Behind the tip of the trailing dislocation 2 a reaction with the leading dislocation I takes place:

$$
\frac{a}{2}[110]+\frac{a}{2}[101] \rightarrow \frac{a}{3}[211]+\operatorname{SSF}+\frac{a}{6}[211]
$$

The partial dislocation $a / 3[211]$ (3) cuts into the $\gamma$-particle and generates a superlattice stacking fault (SSF), while the second partial dislocation $a / 6[211]$ (4) remains in the $\gamma / \gamma$ interface. As can be seen from Fig. 3b, the cutting into the $\gamma$-particles takes places periodically along the dislocation line where the matrix dislocations encounter a $\gamma$-cube. This mechanism is therefore very effective to generate stacking faults. A similar mechanism has been observed by FellerKniepmeier and Kuttner in [011] samples of SRR 99 tested at $1033 \mathrm{~K}$ and $680 \mathrm{MPa}$ [9]. The characteristic feature of this mechanism is the reaction of two coplanar matrix dislocations with different Burgers vectors. This condition would require the operation of two dislocation sources on the same $\{111\}$ plane, which seems quite unlikely at least in the early stages of deformation. In [011] orientation this obstacle can be overcome by cross slip of interfacial dislocations with screw character on $[001]$ planes toward the immobile second interfacial dislocation with $60^{\circ}$ character [9]. whereas in [001] orientations all interfacial dislocations in the predominantly deformed $(001)$ horizontal channel have $60^{\circ}$ character $[10]$. Therefore the easier generation of stacking faults in $[011]$ orientation appears to be a major cause for the pronounced primary creep and the poor strain-lifetimes compared to [001].

The deformation of the matrix-phase occurs independent of orientation by the well know mechanism of the expansion of $a / 2<110>\{111\}$ dislocation loops on $\{111\}$ slip planes in the narrow matrix channels $[5,12,13]$ (see Fig. 4). The propagation of dislocation loops starting from grown-in sources is directed by the effective shear stresses in the matrix channels, which vary with the orientation of the matrix channels with respect to the load axis. The effective stress level in a matrix channel is determined by the superposition of external load and internal coherency stresses. In [001] orientation this effect leads to a stress concentration in horizontal matrix channels and a reduced stress level in vertical channels $[5,10,14]$. In contrast to that in [01I] orientation two types of matrix channels, the so called "roof channels", which are inclined at an angle of $45^{\circ}$ to the stress axis, are highly stressed and only the (100) "gable channels" aligned parallel to the load axis experience lower stresses [9]. For the highly symmetric [T11] orientation all three types of matrix channels are equally stressed.

In the absence of intense $\gamma$-particle cutting primary creep in $[001]$ and $[\overline{1} 11]$ orientation at $500 \mathrm{MPa}$ is controlled by matrix deformation. In [00I] orientation the plastic deformation of the matrix phase is confined to the highly stressed horizontal matrix channels. The activation of multiple $<110>\{111\}$ slip systems results in a rapid build-up of dense dislocation networks in the $\gamma^{\prime} \gamma^{\prime}$ interfaces, which inhibit further deformation. Accordingly the primary creep stage is completed after small strains in crystals oriented near [001]. The pronounced primary creep in sample B with a large misorientation of $13^{\circ}$ towards $[111]$ was caused by coplanar slip on the ( $\overline{1} 11)$ plane.

In $[\overline{1} 11]$ the multiplicity of $\langle 110\rangle\{111\}$ slip systems and the low value of the Schmid factor for octahedral slip of 0.27 should result in a homogenous matrix deformation and superior creep strength similar to [001]. The actual primary creep rates, however, are more similar to those of [011] oriented crystals, where primary creep deformation occurs in a much more heterogeneous way. Poor creep strength of [T11] oriented crystals was reported by Caron et al. [2] for several alloys containing large $\gamma$-precipitates, which promoted homogenous matrix deformation instead of the heterogeneous shearing of the $\gamma$-phase by $\{111\}<112>$ slip observed for small $\gamma$-particles. The reasons for the negative effect of homogenous matrix deformation lie in the influence of coherency stresses on dislocation movements. TEM 

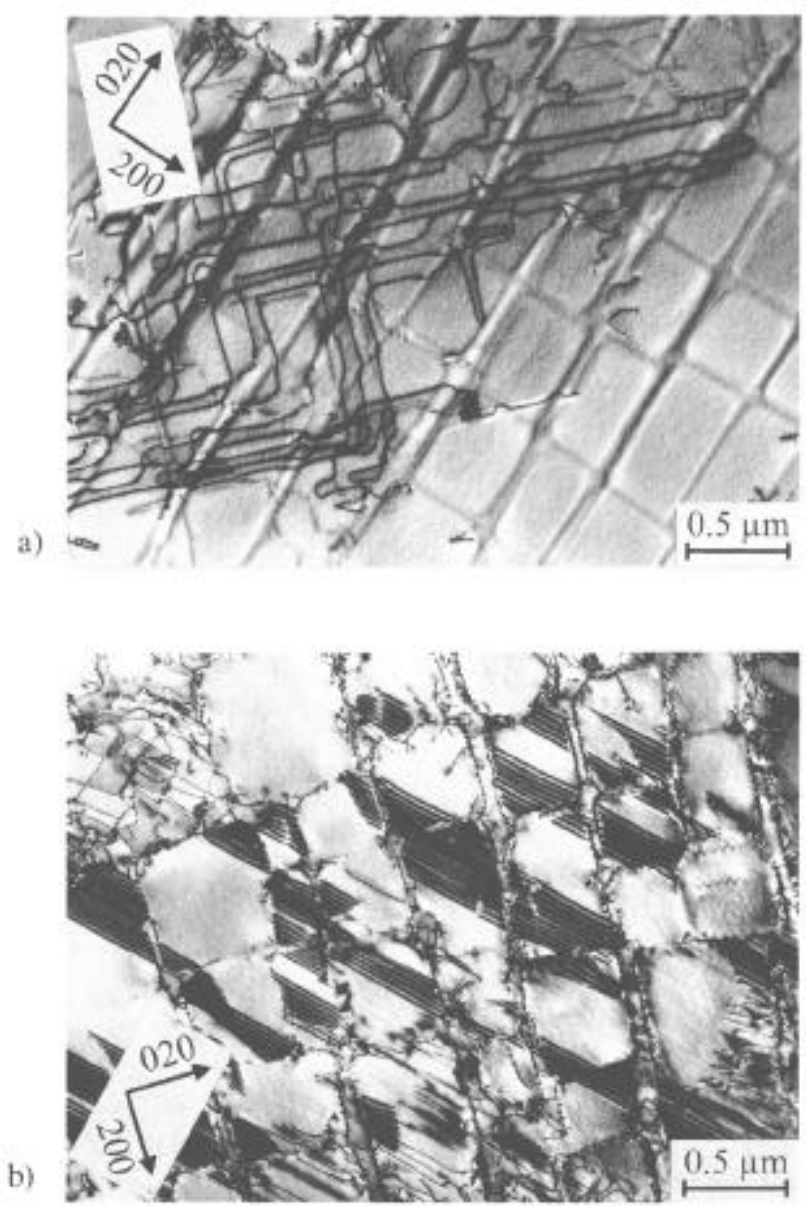
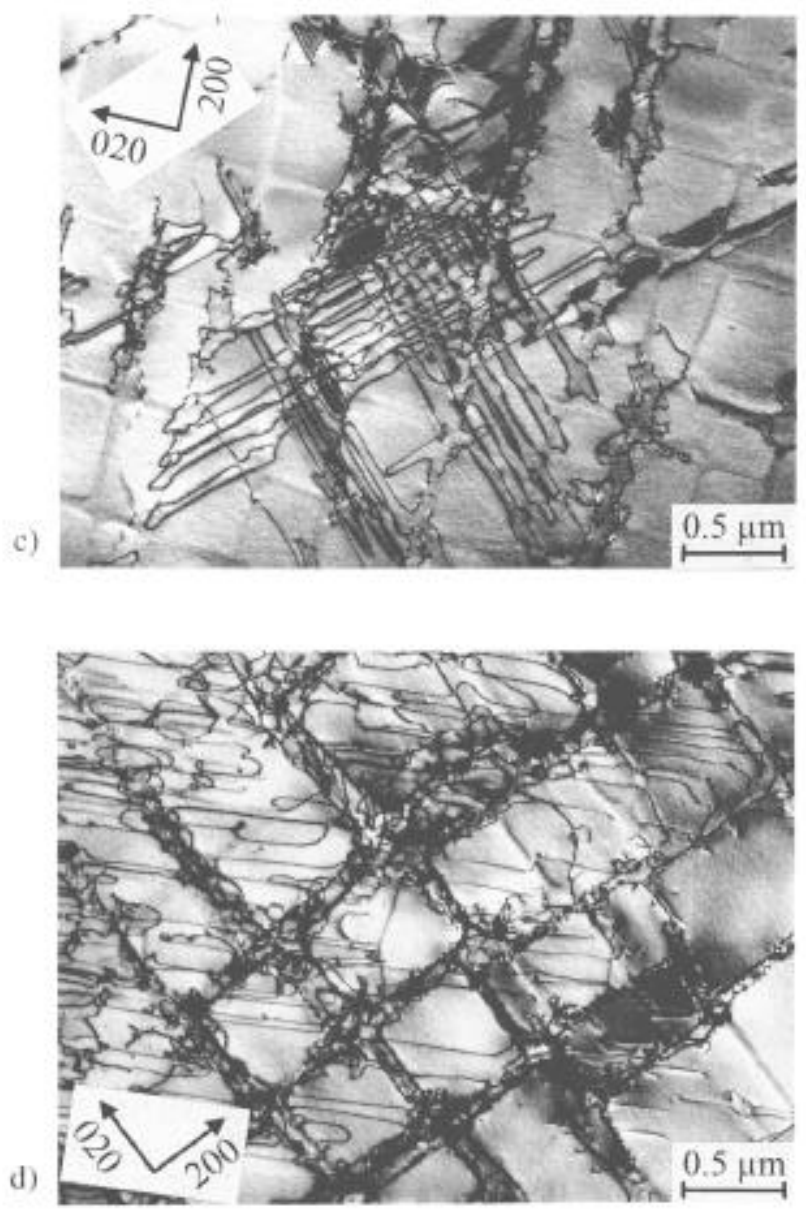

Figure 4: Typical microstructures of CMSX-4 single crystals in the primary creep stage after testing at $\mathrm{T}=1123 \mathrm{~K}$ and $\sigma=500 \mathrm{MPa}$; a) $[001]$ orientation: Expansion of dislocation loops in a horizontal $(001)$ matrix channel $(0.14 \%$ strain). b) [011] orientation: Sample oriented for coplanar slip on the (111) plane. The $\gamma$-phase is cut by extended bands of stacking faults $(1.8 \%$ strain), c) [011] orientation: Intersecting slip in a sample oriented for slip on two $\{111\}$ planes $(0.5 \%$ strain). d) [111] orientation: Interfacial dislocation segments with predominantly parallel line vectors $(0,14 \%$ strain).

analysis of the dislocation structures in deformed [ 111$]$ samples revealed a selection mechanism for matrix dislocations caused by coherency stresses. In each channel type four $\{111\}<110>$ slip systems on two slip planes are active, producing dislocations with either $60^{\circ}$ or

\section{\{111\} Slip planes}

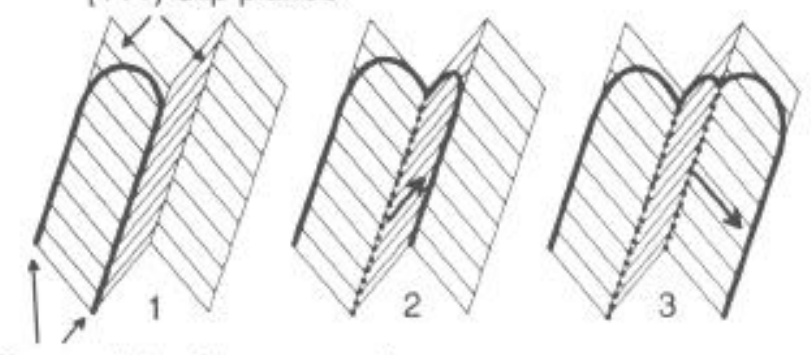

\section{Screw dislocation segments}

Figure 5: Schematic sketch of repeated cross slip of interfacial screw dislocation in [111] orientation. predominantly screw character [15]. Due to the crystallography in [ $\overline{1} 11]$ orientation the line vectors of the dislocations in a given channel type are parallel as can be seen from Fig. 4c. Accordingly the probability of dislocation interaction is low and strain hardening is poor despite the operation of multiple slip systems.

Repeated cross slip of interfacial screw dislocations [15], which were produced by the usual Orowan by-passing mechanism, on two $\{111\}$ planes was also observed exclusively in $[\overline{1} 11]$ orientation as schematically shown in Fig. 5. An expanding dislocation loop in a matrix channel deposits long segments with screw character in the $\gamma / \gamma$ interfaces. The long screw segments are glissile by cross slip to a second $\{111\}$-type slip plane. This mechanism is possible due to equal shear stresses on the two possible \{111\} slip planes of the screw dislocation segments and explains the high secondary creep rates typical for [111]. The macroscopic deformation associated with this mechanism is identical to that of $\{100\}<110>$ cube slip. 
This may explain, why Pan et al. [16] found changes in the shape of cross sections of creep deformed SRR 99 single crystals in $[\bar{T} 11]$ orientation, which are only compatible with deformation on cubic slip systems.

\section{Secondary Creep at $1123 \mathrm{~K}$}

The secondary creep rates, which mainly control the total rupture lives, are much less sensitive to small misorientations than the primary creep behavior. Regardless of orientation and stress deformation occurs mainly in the matrix phase, even in samples with prominent cutting of the $\gamma$-phase by stacking faults during primary creep. TEM investigations on [001] oriented samples revealed, that the number of stacking faults remains virtually constant after the maximum of the creep rate up to strains of about $6 \%$. The depletion of the stacking fault mechanism with increasing strain can be explained by the loss of coherency between the two phases caused by interfacial dislocation networks [17]. Coherency stresses were shown to facilitate the dissociation of matrix dislocations required for the generation of stacking faults. With increasing strain cutting of the $\gamma^{\prime}$-phase by individual pairs of $a / 2<110>(111)$ dislocation pairs is observed. Pollock and Argon [11] related this behavior to the build-up of stresses in the $\gamma^{\prime}$-particles caused by the plastic flow of the matrix phase between the undeformable $\gamma$-cubes.

The rate-controlling mechanism, however, is the deformation of the matrix phase. TEM observation showed for samples of all orientations a quasi-stationary dislocation structure, which is retained in the strain range investigated in this study. This dislocation structure still reflects the influence of coherency stresses discussed above.

In [001] specimens the dislocation density in horizontal matrix channels is still considerably higher than in vertical channels. The concentration of matrix deformation on horizontal matrix channels contributes significantly to the superior creep resistance of the [001] orientation. Other beneficial features are the multitude of active $\{111\}<110\rangle$ type slip systems and the high stresses required for the dislocation loop process in the narrow matrix channels. The outcome of these processes is a quick increase in dislocation density after small strains and the build-up of dense dislocation networks in the $\gamma / \gamma$-interfaces.

In [011] orientation the creep resistance of the matrix phase is clearly weaker than for [001]. Here two types of matrix channels, the so called "roof channels" are highly stressed and therefore subjected to strong deformation. The number of active slip systems is only four instead of eight for [001] and from Schmid factor considerations no cross slip on [111] planes is possible. Therefore the probability of dislocation interactions with a hardening effect is reduced. The lower resistance to matrix deformation together with the pronounced $\gamma^{\prime}$-particle shearing result in higher secondary creep rates as compared to the $[001]$ orientation.

In [T11] orientation the creep curves (see Fig. la) show no clear transition from primary to secondary creep stage. Since deformation in the matrix phase occurs by $\{111\}<110\rangle$ multiple slip already during primary creep, the amount of strain hardening following the maximum of the creep rate in specimen F is rather limited (see Fig. la), resulting in the highest secondary creep rates of all orientations. TEM observations showed, that the basic deformation mechanisms are the same during primary and secondary creep [17]. Therefore the poor creep strength of [111] oriented crystals can be understood as a combined effect of the uniform matrix deformation in all three types of matrix channels, poot strain hardening due to a selection mechanism for active slip systems in each channel type and the high mobility of interfacial screw dislocations by repeated cross slip on $\{111\}$ planes. a)

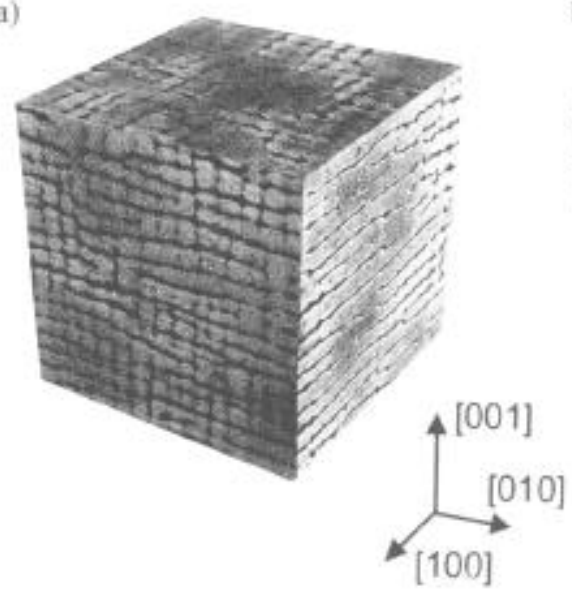

b)

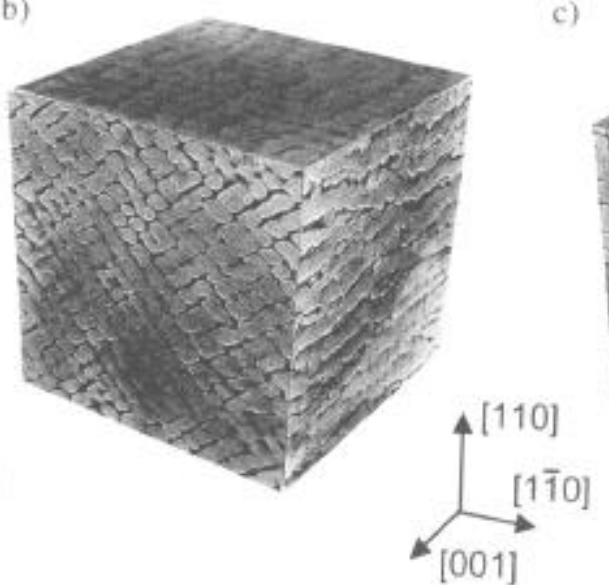

c)

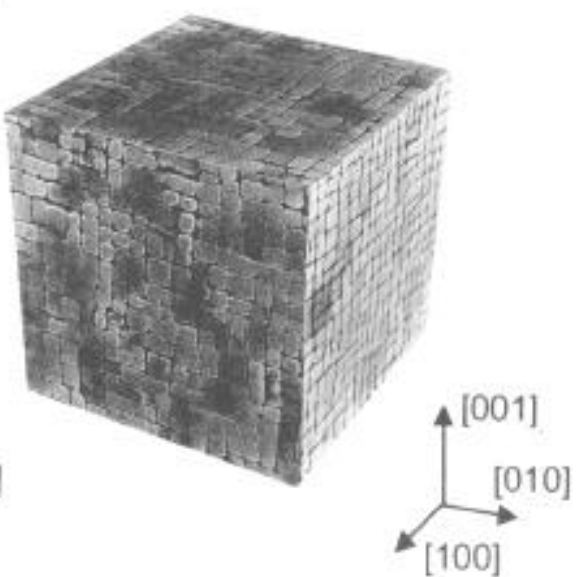

Figure 6: Morphological changes of the $\gamma^{\prime}$-phase (light color) in creep deformed CMSX-4 crystals tested at $\mathrm{T}=1253 \mathrm{~K}$ and $\sigma=350 \mathrm{MPa}$. SEM micrographs taken from three cuts perpendicular to each other were arranged in order to provide a 3-dimensional view of the $\gamma^{\prime}$-structure. a) After creep testing in [001] orientation for 28h. b) After creep testing in [011] direction for $28 \mathrm{~h}$. c) After creep testing in [111] orientation for $4 \mathrm{~h}$. 


\section{Creep at $1253 \mathrm{~K}$}

The main features of creep deformation of superalloys at high temperatures are the instability of the $\gamma^{\prime}$-morphology and a homogenous character of plastic deformation due to the absence of extended stacking fault bands and a higher mobility of dislocations. A comprehensive study on the creep behavior of several alloys by Caron et al. [2] suggests, that these effects reduce the orientation dependence of creep strength. This tendency is also reflected in the creep curves of [001] and [011] oriented CMSX-4 single crystals (see Fig. 1b). The primary creep stage, which showed the highest degree of anisotropy at lower temperatures, is here reduced to a short transient. In the following distinct minimum of the creep rate a quasi-steady state is reached. It should be noted, that in the representation of creep curves as creep rate vs. strain plots sections with low strain rates appear compressed. Actually the specimens spend a great fraction of their total lifetime in this stage. The minimum creep rates appear to be quite independent of orientation, although plastic deformation is concentrated in highly stressed matrix channels in the same way as described above for lower temperatures. The effect of coherency stresses is here aggravated by the gradual process of directional coarsening of the $\gamma$-phase, which results in the disappearance of lower stressed matrix channels.

The evolution of the $\gamma^{\prime}$-morphology results in characteristic spatial arrangements of the $\gamma^{\prime}$-phase shown in Fig. 6. The initially cuboidal $\gamma$-precipitates form rafts perpendicular to the load axis in [001] orientation and bars aligned parallel to the [100] direction for [011] oriented samples. This process is almost completed in the samples deformed to about $4 \%$ strain. The evolution of the $\gamma^{\prime}$-morphology corresponds with a drastic reduction of creep strength. The reasons for this are the broadening of the remaining matrix channels and a reduction of the threshold stress for the cutting of the $\gamma^{\prime}$ phase by a/2<110>\{111\} dislocation pairs [18].

In [111] orientation the typical shape of the creep curves with a pronounced maximum of the creep rate is retained up to high temperatures and results in very short lifetimes for small strains. The microstructure in the primary creep stage, where the initial cuboidal $\gamma^{\prime}$-morphology is still intact, shows the same features as at lower temperatures, i.e. homogenous deformation in all types of matrix channels, cross slip of screw dislocations and a reduced number of active slip systems. In the following secondary creep stage the $\gamma^{\prime}$-particles coarsen irregularly parallel to the cubic directions. Due to the high creep rates in [ 111 ] the extent of particle coarsening after equal strains cannot be easily compared to other orientations due to the large differences in creep test duration. Careful inspection of the creep curves of [ $\overline{1} 11]$ oriented crystals (see Fig. 1b), however, revealed, that the creep rates are still decreasing at strains as large as $5 \%$. This indicates, that the undirected coarsening of the $\gamma^{\prime}$ phase in [ $\overline{1} 11]$ orientation tends to promote a reduction of creep rates rather than the intense softening associated with the morphological changes typical for [001] and [011] orientations. The reasons for this unusual behavior are not clear and require further investigations.

\section{Conclusions}

Tensile creep tests of CMSX-4 single crystals at temperatures of $1123 \mathrm{~K}$ and $1253 \mathrm{~K}$ showed a high degree of anisotropy, which is most pronounced in the primary creep stage.

At $1123 \mathrm{~K}$ creep lifetimes for small strains of 0.5 and $2.0 \%$ decrease drastically in the order [001] - [011] - [T11]. For most orientations plastic deformation occurs predominantly in the matrix phase and is strongly influenced by internal coherency stresses, which control the fraction of deformable matrix volume and lead to deviations from Schmid's law for the active slip systems. Extensive cutting of the $\gamma^{\prime}$-phase by $\{111\}<112>$ slip is observed only during primary creep of [011] oriented crystals and for [001] orientation under the highest level of applied stress. In these cases a pronounced primary creep stage and a high sensitivity of primary crcep behavior to small misorientations are observed.

At $1253 \mathrm{~K}$ the creep anisotropy is generally reduced and creep of [001] and [011] oriented crystals is controlled by the instability of the $\gamma^{\prime}$-morphology. The creep strength of the $[\overline{1} 11]$ orientation remains poor.

\section{References}

1. R.A. MacKay and R.D. Maier, "The Influence of Orientation on the Stress Rupture Properties of Nickel-Base Superalloy Single Crystals", Metall. Trans., 13A (1982), 1747-1754.

2. P. Caron, Y. Ohta, Y.G. Nakagawa and T. Khan, "Creep Deformation Anisotropy in Single Crystal Superalloys", in: Superalloys 1988, eds. S. Reichmann et al. (Warrendale, PA: The Metall. Society of AIME, 1988), 215224.

3. A.A. Hopgood and J.W. Martin, "The Creep Behaviour of a Nickel-Based Single Crystal Superalloy", Mater. Sci. Engng., 82 (1986), 27-36.

4. V. Sass, W. Schneider and H. Mughrabi, "On the Orientation Dependence of the Intermediate Temperature Creep Behaviour of a Monocrystalline Nickel-Base Superalloy", Scripta metall. mater., 31 (1994), 885-890.

5. D.J. Frazier, J.R. Whetstone, K. Harris, G.L. Erickson and R.E. Schwer, "Process and Alloy Optimization for CMSX-4 Superalloy Single Crystal Airfoils", Cost Conf. Liège, Sept. 24.-27. 1990, Proc. Part II, 1281-1300.

6. D. Blavette, P. Caron and T. Khan, "An Atom-Probe Study of Some Fine Scale Microstructural Features in NiBased Single Crystal Superalloys", in: Superalloys 1988, 
eds. S. Reichmann et al. (Warrendale, PA: The Metall. Society of AIME, 1988), 305-314.

7. U. Glatzel, "Microstructure and Internal Strains of Undeformed and Creep Deformed Samples of a Nickel-Base Superalloy" (Habilitation thesis, Technical University Bcrlin, 1994).

8. H. Mughrabi, W. Schneider, V. Sass and C. Lang, "The Effect of Raft Formation on the High Temperature Creep Deformation Behaviour of the Monocrystalline Nickel-Base Superalloy CMSX-4", in: Strength of Materials, Proc. ICSMA-10, eds. H. Oikawa et al., The Japan Institute of Metals, (1994), 705-708.

9. M. Feller-Kniepmeier and T. Kuttner, "[011] Creep in a Single Crystal Nickel Base Superalloy at 1033K", Acta metall. mater., 42 (1994), 3167-3174.

10. M. Feller-Kniepmeier, U. Hemmersmeier, T. Kuttner and T. Link, "Analysis of Interfacial Dislocations in a Single Crystal Nickel-Base Superalloy after [001] Creep at 1033K. Evolution of Internal Stresses", Scripta metall. mater., 30 (1994), 1275-1280.

11. T.M. Pollock and A.S. Argon, "Creep Resistance of CMSX-3 Nickel Base Superalloy Single Crystals", Acta metall. mater., 40 (1992), 1-30.

12. A. Fredholm and J.L. Strudel, "On the Creep Resistance of some Nickel Base Single Crystals", in: Superalloys 1984, Proc. 5th Int. Symp. on Superalloys, eds. M. Gell et al, (Warrendale, PA: The Metall. Society of AIME, 1984), 211-220.

13. T. Link and M. Feller-Kniepmeier, "Elektronenmikroskopische Untersuchungen von $\gamma / \gamma^{\prime}$-Phasengrencen in der einkristallinen Nickelbasislegierung SRR 99 nach Hochtemperaturkriechen", Z. Metallkunde, 79 (1988), 381 387.

14. L. Mueller, U. Glatzel and M. Feller-Kniepmeier, "Calculation of the Internal Stresses and Strains in the Microstructure of a Single Crystal Nickel-Base Superalloy During Creep", Acta metall. mater., 41 (1993), 3401-3411.

15. R. Voelkl, U. Glatzel and M. Feller-Kniepmeier, "Analysis of Matrix and Interfacial Dislocations in the Nickel-Base Superalloy CMSX-4 after Creep in [111] Direction", Scripta metall. mater., 31 (1994), 1481-1486.

16. L.-M. Pan, L. Scheibli, M.B. Henderson, B.A. Shollock and M. McLean, "Asymmetric Creep Deformation of a Single Crystal Superalloy”, Acta metall. mater., 43 (1995), 1375-1384.
17. T. Link and M. Feller-Kniepmeier, "Shear mechanisms of the $\gamma^{\prime}$-Phase in Single Crystal Superalloys and their Relation to Creep", Met. Trans., 23A (1992), 99-105.

18. P. Caron, P.J. Henderson, T. Khan and M. McLean, "On the Effects of Heat Treatment on the Creep Behaviour of a Single Crystal Superalloy", Scripta metall. mater., 20 (1986), 875-880. 